\title{
Implications of a Negative Sentinel Node on Radiation Field Design for Chest Wall Recurrences
}

\author{
Bruce G. Haffty, MD \\ Department of Radiation Oncology, Rutgers Cancer Institute of New Jersey, Robert Wood Johnson Medical School and \\ New Jersey Medical School, New Brunswick, NJ
}

Locoregional management of breast cancer is a rapidly evolving and complex issue that requires input from all members of the multidisciplinary team caring for the patient. Surgical approach, timing of reconstruction, timing and sequence of systemic therapy, timing of radiation, and radiation field design all rely on each other, requiring close coordination and communication among the multidisciplinary team. Management of the patient with a local or regional recurrence is even more complicated, as there are fewer data, trials, and guidelines upon which to base clinical decision making. The clinician and patient are often faced with decision making that is based on clinical judgment, retrospective data, training, experience, and often extrapolation from initial management where there is a larger body of evidence-based data.

Sentinel node mapping and sampling has clearly had a major impact in both the surgical and radiotherapeutic management of breast cancer. ${ }^{1-3}$ For patients presenting with early stage disease, a negative sentinel node biopsy finding in the primary management of breast cancer has become an accepted standard replacing full axillary dissection, and it has clearly affected the quality of life of patients with breast cancer. ${ }^{1}$ However, there remain controversies regarding management of a negative sentinel node after neoadjuvant systemic therapy. ${ }^{4}$ Management of the patient with a positive sentinel node remains variable, depending on a number of clinical, pathologic, and risk factors, as well as physician and patient preferences. ${ }^{3,5,6}$ Options range from completion axillary dissection, with

(C) Society of Surgical Oncology 2015

First Received: 27 October 2015;

Published Online: 17 November 2015

B. G. Haffty, MD

e-mail: hafftybg@cinj.rutgers.edu radiation field design dependent on the result of the dissection; comprehensive regional nodal irradiation without further dissection; or no further treatment beyond radiation to the intact breast, based on the results of ACOSOG Z0011. ${ }^{2,5,6}$ Randomized data from AMAROS suggest that radiation provided to the regional nodes is as effective as surgery, with lower lymphedema rates compared to axillary dissection in the setting of a positive sentinel node at initial diagnosis. ${ }^{6}$ The ongoing Alliance A011202 trial is evaluating the role of axillary radiation compared to axillary dissection for a persistently positive sentinel node after neoadjuvant systemic therapy.

The role of sentinel node mapping and sampling at the time of locoregional recurrence, particularly for postmastectomy chest wall recurrence, and its impact on radiotherapy design remains an understudied area, with wide variability in practice. Given the paucity of data, there are no clear guidelines or preferred approaches, leaving clinicians to rely on reports, such as the one by Johnson et al. that accompanies this editorial. ${ }^{7}$ The authors clearly document the historical literature, rationale for this approach, and the details of the surgical, radiotherapeutic, and systemic management of the patients in the series. Although the interpretation is limited by its retrospective design, limited follow-up, and relatively small patient numbers and events, the study confirms the feasibility of sentinel node sampling in the setting of an isolated chest wall recurrence in those patients who previously were managed with a sentinel node at initial diagnosis. Furthermore, the authors have demonstrated a low rate of regional failure using the results of the sentinel node procedure to direct radiotherapy fields. Specifically, in the setting of a successful negative sentinel node, radiation was directed at the chest wall only, avoiding supraclavicular, axillary, and internal mammary radiation. It should be emphasized, however, that it appears that a successful 
negative axillary sentinel node with chest-wall-only directed radiation was used in only seven patients, one of whom experienced an internal mammary recurrence. To date, the authors have not reported any out-of-field supraclavicular relapses, and they suggest that in the setting of successful mapping of a negative axillary sentinel node at the time of chest wall recurrence, radiotherapy directed at the chest wall only may decrease treatment related morbidity without compromising locoregional control.

Although the authors have carefully documented their experience, given the limited numbers of patients and events, omission of radiation to the regional nodes in the setting of a chest wall recurrence should be approached with caution. The breast cancer patient who experiences isolated locoregional recurrence without evidence of distant metastasis can, with appropriate local, regional, and systemic therapy, enjoy a long disease-free survival and excellent quality of life. ${ }^{8,9}$ My own bias, which is based on a number of factors, including the chance of regional repeat recurrence, albeit relatively small, in an adjacent nodal basin, and to some extent extrapolating from trials demonstrating benefits of regional nodal irradiation in the primary treatment of high-risk breast cancer, is to approach patients with an isolated recurrence with aggressive comprehensive locoregional treatment and appropriate systemic therapy. ${ }^{10-14}$

Patients with an isolated postmastectomy chest wall recurrence represent a high-risk population with respect to overall disease-free survival, distant metastasis, and further locoregional progression. ${ }^{8,15,16}$ Although the risk of subsequent recurrence is high, the patient with an isolated local chest wall relapse does present with a second chance at long-term disease-free survival and cure. In the absence of definitive prospective data to guide therapy, one must rely on both well-described retrospective series, such as the one accompanying this editorial, and on extrapolation from the primary treatment of breast cancer. As Johnson et al. point out, they were able to successfully identify a negative sentinel node at the time of chest wall recurrence in a limited population, and they reported a low regional failure rate when they followed a general policy of sentinel nodedirected radiation fields.

This is a valuable contribution. I concur with the authors that this approach deserves further investigation. Until further data are available, however, my own approach would be, even in the setting of a negative sentinel node or negative axillary sampling at the time of chest wall recurrence, to irradiate the chest wall, undissected axilla, and internal mammary fields. My rationale for this approach is based in part on the probability of a second locoregional relapse in an adjacent untreated nodal basin. In the series accompanying this editorial, $1(14 \%)$ of 7 of the patients failed in the adjacent internal mammary basin, and in the Halverson series, $16 \%$ failed in the adjacent untreated supraclavicular region. ${ }^{10}$ In addition, there is a broad body of evidence-based literature in the primary management of breast cancer suggesting a benefit to regional nodal irradiation in patients with positive nodes, and even in patients with high-risk node-negative disease. ${ }^{11,13,14}$ The recent EORTC randomized trial of chest wall compared to chest wall and regional nodal irradiation demonstrated a benefit to regional nodal irradiation in patients with central or medial node-negative disease. ${ }^{13}$ The benefit extends beyond locoregional control to breast cancer mortality and distant metastasis. Acknowledging the limitation of extrapolating from this body of literature in the primary management to the patient with isolated chest wall recurrence, I consider the patient with an isolated chest wall recurrence to be in a high-risk pool of patients, and in the absence of other available data, I would offer the potential benefit of regional nodal irradiation to this group of patients.

As the field progresses with improvements in prognostic factors, including molecular profiling, advances in imaging, and further studies and follow-up, such as the series reported by Johnson et al., we may move further toward a sentinel node-directed radiotherapy field design in the setting of chest wall recurrence. Although I concur with the authors that further investigation of this approach is warranted, my bias is to continue to offer patients with isolated chest wall recurrences comprehensive chest wall and regional nodal irradiation.

\section{REFERENCES}

1. Lyman GH, Temin S, Edge SB, et al. Sentinel lymph node biopsy for patients with early-stage breast cancer: American Society of Clinical Oncology clinical practice guideline update. $J$ Clin Oncol. 2014;32:1365-83.

2. Giuliano AE, Hunt KK, Ballman KV, et al. Axillary dissection vs no axillary dissection in women with invasive breast cancer and sentinel node metastasis: a randomized clinical trial. JAMA. 2011;305:569-75.

3. Haffty BG, Hunt KK, Harris JR, Buchholz TA. Positive sentinel nodes without axillary dissection: implications for the radiation oncologist. J Clin Oncol. 2011;29:4479-81.

4. Boughey JC, Suman VJ, Mittendorf EA, et al. Sentinel lymph node surgery after neoadjuvant chemotherapy in patients with node-positive breast cancer: the ACOSOG Z1071 (Alliance) clinical trial. JAMA. 2013;310:1455-61.

5. Jagsi R, Chadha M, Moni J, et al. Radiation field design in the ACOSOG Z0011 (Alliance) trial. J Clin Oncol. 2014;32:3600-6.

6. Donker M, van Tienhoven G, Straver ME, et al. Radiotherapy or surgery of the axilla after a positive sentinel node in breast cancer (EORTC 10981-22023 AMAROS): a randomised, multicentre, open-label, phase 3 non-inferiority trial. Lancet Oncol. 2014;15: 1303-10.

7. Johnson JEL, Ewing C, Alvarado M, Park C, Fowble B. Sentinel lymph node mapping in post mastectomy chest wall recurrences: influence on radiation treatment fields and outcome [editorial]. Ann Surg Oncol. 2015. 
8. Aebi S, Gelber S, Anderson SJ, et al. Chemotherapy for isolated locoregional recurrence of breast cancer (CALOR): a randomised trial. Lancet Oncol. 2014;15:156-63.

9. Borner M, Bacchi M, Goldhirsch A, et al. First isolated locoregional recurrence following mastectomy for breast cancer: results of a phase III multicenter study comparing systemic treatment with observation after excision and radiation. Swiss Group for Clinical Cancer Research. J Clin Oncol. 1994;12:2071-7.

10. Halverson KJ, Perez CA, Kuske RR, Garcia DM, Simpson JR, Fineberg B. Isolated local-regional recurrence of breast cancer following mastectomy: radiotherapeutic management. Int $J$ Radiat Oncol Biol Phys. 1990;19:851-8.

11. EBCTCG (Early Breast Cancer Trialists' Collaborative Group), McGale P, Taylor C, et al. Effect of radiotherapy after mastectomy and axillary surgery on 10-year recurrence and 20-year breast cancer mortality: meta-analysis of individual patient data for 8135 women in 22 randomised trials. Lancet. 2014;383:212735.

12. Poortmans P. Postmastectomy radiation in breast cancer with one to three involved lymph nodes: ending the debate. Lancet. 2014;383:2104-6.

13. Poortmans PM, Collette S, Kirkove C, et al. Internal mammary and medial supraclavicular irradiation in breast cancer. $N$ Engl $J$ Med. 2015;373:317-27.

14. Whelan TJ, Olivotto IA, Parulekar WR, et al. Regional nodal irradiation in early-stage breast cancer. N Engl J Med. 2015;373:307-16.

15. Freedman GM, Fowble BL. Local recurrence after mastectomy or breast-conserving surgery and radiation. Oncology (Williston Park). 2000;14:1561-81.

16. Haffty BG, Hauser A, Choi DH, et al. Molecular markers for prognosis after isolated postmastectomy chest wall recurrence. Cancer. 2004;100:252-63. 